Portland State University

PDXScholar

\title{
Effects of Hormone Replacement Therapy (HRT) on Gene Expression in the Amyloid Processing Pathway of Ovariectomized Rhesus Macaque Females Under an Obesogenic Diet
}

\author{
Megan Ball \\ Portland State University
}

Follow this and additional works at: https://pdxscholar.library.pdx.edu/honorstheses

Part of the Genetics and Genomics Commons, Hormones, Hormone Substitutes, and Hormone Antagonists Commons, Physiological Processes Commons, and the Women's Health Commons Let us know how access to this document benefits you.

\section{Recommended Citation}

Ball, Megan, "Effects of Hormone Replacement Therapy (HRT) on Gene Expression in the Amyloid Processing Pathway of Ovariectomized Rhesus Macaque Females Under an Obesogenic Diet" (2021). University Honors Theses. Paper 1153.

https://doi.org/10.15760/honors.1189

This Thesis is brought to you for free and open access. It has been accepted for inclusion in University Honors Theses by an authorized administrator of PDXScholar. Please contact us if we can make this document more accessible: pdxscholar@pdx.edu. 
Effects of hormone replacement therapy (HRT) on gene expression in the amyloid processing pathway of ovariectomized rhesus macaque females under an obesogenic diet

\author{
by \\ Megan Ball \\ An undergraduate honors thesis submitted in partial fulfillment of the \\ requirements for the degree of \\ Bachelor of Science \\ in \\ University Honors \\ and \\ Biology
}

Thesis Adviser

Rita Cervera-Juanes

Portland State University 


\section{Abstract}

Estrogens rapidly decline at the onset of menopause, putting women at higher risk of osteoporosis, obesity, and neurodegenerative diseases, including Alzheimer's disease (AD). Estradiol hormone therapies (HT) have been suggested to limit these negative effects on women, with controversial findings as to their effectiveness. A study at OHSU recently found differentially expressed genes (DEGs) using RNAseq between ovariectomized/hysterectomized $(\mathrm{OvH})$ old (range $=19.4-23.2$ years) female rhesus macaques on HT and those on a placebo on chronic western style diet (WSD). The DEGs identified in the amyloid processing pathway of the amygdala were selected for validation using RT-qPCR because $\beta$-amyloid peptide (A $\beta)$, made in the amyloid processing pathway, has a strong correlation with AD risk. The selected genes were: AKT3, APP, CAPN1, CAPNS1, CSNK2A1, CSNK2A2, MAPK1, MAPK14, MAPT, MARK1, NCSTN, PRKAR1A, PRKAR2A, PRKAR2B, and PRKCE. All the genes tested were found to be significantly downregulated $(\mathrm{p}<0.05)$ in the OvH-HT females. These genes were also tested in another set of old and young female rhesus macaques on a standard diet (SD) and were not differentially expressed using qPCR. Since the RNAseq analysis used a cutoff of $2.3 \log$ CPM to select genes, the qPCR tests confirming the DEGs at this level tells us $2.3 \log \mathrm{CPM}$ is a valid cutoff to use in future RNAseq analysis. The genes in the amyloid processing pathway being significantly downregulated with HT suggests beneficial effects of the HT with WSD because this pathway regulates $A \beta$ and accumulation of $A \beta$ plaques in the brain are a hallmark of $A D$.

\section{Introduction}

The importance of estrogens during menopause

Each year in the United States, approximately 1.3 million women become menopausal. Menopause is physiological status characterized by the non-pathological cessation of menses for 
at least 12 months as a result of estrogen deficiency, which occurs naturally in human females during mid to late adulthood [1]. Estradiol is an estrogen that is a key hormone involved in many biological functions through complex processes like those of thermoregulation, circadian pathways, and neuroendocrine pathways [2-5]. Upon the loss of estradiol in menopause, many of the processes that estrogen once regulated begin altering, leading to adverse physiological effects. For instance, menopause has been liked to osteoporosis, which affects more than 250,000 menopausal and postmenopausal women, higher rates of coronary heart disease [1], and psychiatric disorders like sleep disruptions, memory loss, and mood disorders [6]. Given the critical and diverse physiological roles of estradiol, maintaining estrogen levels close to the premenopause levels has been the gold standard for preventing menopause-related health issues.

\section{Menopause and hormonal replacement therapy}

Women are almost two times more at risk of developing Alzheimer's Disease (AD) as men, which is not fully explained by lifespan differences between sexes [7-8]. This risk is even higher in postmenopausal women, which lead to establishing a link between menopause and cognitive decline $[5,9]$. This cognitive decline can include psychological impacts like depression, anxiety, and neurodegenerative diseases [10].

As a preventative measure to offset these potential negative effects of menopause, estradiol hormone therapies (HT) have been traditionally prescribed to women in menopause to reduce risk of $\mathrm{AD}$ and mild cognitive impairment through the menopausal transition [11]. Results from such HT studies are controversial in their findings. Studies from the Women's Health Initiative estimated overall health risks to be greater than the benefits, with increases in risk of stroke, breast cancer and cardiovascular disease [7-8, 10, 12]. Meanwhile, other studies have found benefits of HT with improved psychological well-being and self-esteem, decreased 
adipose tissue, fasting serum glucose and insulin levels, and decreased bone density loss [13-15]. It has been suggested that the positive effects may be due to administered HT during a particular "window of opportunity," where HT can be given to peri and pre- menopausal women to prevent menopausal-related cognitive decline [10-12, 16]. In animal models and human studies, research has identified the beneficial effects of HT if the therapy is administered at the onset of menopause during this "window of opportunity," [17-22].

\section{Menopause and Obesity}

During estrogen deficiency, metabolic dysfunction is promoted and predisposes women to obesity, metabolic syndrome, and type II diabetes [23], as well as increased bone density loss and cardiovascular risk $[11,24]$. Obesity is a leading cause of morbidity and mortality in the U.S. [25] with $42.4 \%$ of the population considered to be obese in 2018 according to the CDC [26]. Factors such as ethnicity, genetic predisposition and rapid fall of estrogen levels can all contribute to increased body weight [27], along with diet. Western style diet (WSD) is considered to be a major contributor to the obesity epidemic worldwide with high fat and sodium, high calories, and excess sugar [28]. Moreover, obesity has been identified as a major risk factor for cognitive decline and AD development [29]. Given that many women going through menopause experience significant weight gain [30], it remains largely uninvestigated how obesity and estrogen in HT interact [25].

\section{Macaque Model to study menopause and HT}

Rhesus macaques present suitable animal model to study menopause because they have the most robustly studied reproductive senescence of all nonhuman primates and females undergo similar endocrine changes as human females in late adulthood, resembling a menopausal transition [31]. These animals also exhibit similar neuroendocrine changes in the brain as 
humans with age [31]. Furthermore, in aged female rhesus macaque research models, studies showed possible benefits of HT administered to ovariectomized/hysterectomized $(\mathrm{OvH})$ females [17-19]. The benefits observed included reduced anxiety-like behaviors and reduced sedentariness as seen in other females on obesogenic diet [17-22, 32]. Positive memory and cognitive effects have also been seen in aged female OvH rhesus macaques on obesogenic diets, particularly if HT starts immediately after estradiol is removed [33].

\section{Recent Findings}

Because women are at higher risk for neurocognitive diseases during and after menopause and this prevalence is increased in obese and overweight women, the molecular mechanisms behind these changes were looked at further in a recent study at Oregon Health and Science University (OHSU). At OHSU an in-depth transcriptome analysis was performed on brain tissues collected from old OvH female rhesus macaques that received chronic HT while on an obesogenic diet [34]. Aged female rhesus macaques (range $=19.4-23.2$ years) were OvH and kept on a high fat, high sugar Western-style diet (WSD) for 30 months. Half of the females received HT immediately following OvH and half were placebo controls. From RNAseq data, the study identified a total of 6,170 differentially expressed genes (DEGs) among the control and HTtreated groups from these tissues: occipital cortex (OC), prefrontal cortex (PFC), hippocampus (HIP), and amygdala (AMG). These brain regions were chosen because they contribute to cognitive function, learning, and memory [35-40]. Of the 6,170 DEGs identified, the AMG had the most DEGs (4,993). Pathway enrichment analysis found neuroinflammation downregulation in the AMG. The signaling pathways for synaptogenesis, circadian rhythm, mitochondrial dysfunction, mTOR, glutamate, serotonin, GABA, dopamine, noradrenaline/adrenaline, glucocorticoid receptor signaling, neuronal NOS and amyloid processing were exclusively 
enriched in AMG. Taken together, these pathways' gene expression being altered expand the link between cognitive decline and obesity, and that the AMG is impacted by AD progression [34]. The signaling pathways for amyloid processing in the AMG, along with other findings from the study indicate a protective role for estradiol HT to prevent some of the negative health effects of individuals on obesogenic diets going through menopause.

Amyloid Processing as the focus of This Study

This study functions as a follow-up on the Cervera-Juanes et al. paper [34] and continues analysis of the amyloid processing signaling pathway for genes that might be involved in the development of amyloid plaques in $\mathrm{OvH}$ macaques. This pathway was of particular interest because amyloid plaques are a hallmark of $\mathrm{AD}$ [41]. Amyloid plaques are made up of $\beta$-amyloid peptide $(A \beta)$ extracellular deposits in the brain [42]. AD can be genetically inherited in families when mutations in some of the genes for amyloid processing are inherited, such as the APP gene ( $\beta$-amyloid precursor protein), PS1 or PS2 (presinilin-1 or -2) [42]. Alterations in gene expression linked to a chronic obesogenic diet may be linked to the increased risk for $\mathrm{AD}$ in obese and menopausal women.

Using RT-qPCR, this study seeks to validate the RNAseq findings from the CerveraJuanes et al. paper [34] using the same RNA from AMG tissue samples. In the RNAseq study, the cutoff for including genes in the study was $0.2 \mathrm{CPM}$ for AMG, which is approximately 10 reads per minimum library size [43]. A fold change greater than or equal to 1.25 and a p-value less than or equal to 0.05 was used to select DEGs. Given the relaxed cutoff that was used, this study seeks to validate the RNAseq findings within the amyloid pathway using reverse transcription-quantitative polymerase chain reaction (RT-qPCR). Our results provide valuable evidence to confirm the previous RNAseq findings and support a protective relationship of HT 
against alterations in the amyloid pathway in ovariectomized females chronically exposed to an obesogenic diet.

\section{Methods}

These studies and their respective subjects were approved by the Oregon National Primate Research Center (ONPRC) Institutional Animal Care and Use Committee.

\section{Experiment 1 Subjects}

The subjects in this study are the same as those previously described [34] and are comprised of 14 old (range $=19.4-23.2$ years) female rhesus macaques (Macaca mulatta). They were maintained on a WSD (TAD Primate Diet; LabDiet, Inc) for the duration of the study (figure 1). WSD was composed of calories with $36 \%$ fat, $44 \%$ carbohydrates (includes $18.5 \%$ sugars), and $18 \%$ protein [34]. At the 6-week mark into the study, animals were OvH and half began HT (figure 1).

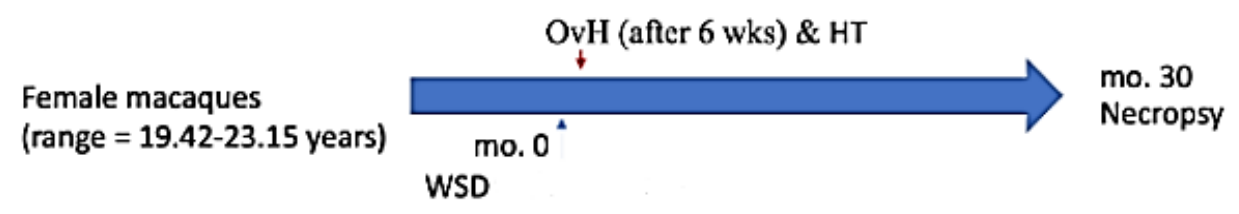

Figure 1 Study design for animals in experiment 1, with WSD introduced at month 0 of study. OvH took place at 6 weeks into the study, and HT began for half $(n=7)$ of the study subjects. Necropsies were performed at the end of the 30-month study period.

\section{Experiment 2 Subjects}

The subjects of this study are comprised of 7 young (range $=9.8-13.9$ years) and 7 old (range $=21.8-26.1$ years) female rhesus macaques (Macaca mulatta). They were housed under the same conditions as previously described [34] with the same daily mealtimes and supplemental foods provided as the animals in experiment 1. For the duration of the study, they were maintained on a standard diet (Monkey Diet, LabDiet, Inc., St Louis, MO, USA) composed 
of calories with $13 \%$ fat, $69 \%$ complex carbohydrates (includes $6 \%$ sugars), and 18\% protein. These subjects were not OvH nor received HT.

\section{Euthanasia}

The same process was used after the 30-month duration of the studies for each experiment as previously described [34]. The euthanasia was performed by trained ONPRC Division of Comparative Medicine Veterinarians following guidelines set forth by the Panel on Euthanasia of the American Veterinary Medical Association. The same methods to isolate AMG tissue were used across all animals as described previously [34].

\section{RNA isolation}

Experiment 1 samples were the same AMG RNA from the Cervera-Juanes et al. paper [34]. RNA was isolated from experiment 1 and 2 AMG tissue samples per the manufacturer's recommendations using the All-Prep DNA/RNA/miRNA Universal Kit (Qiagen Sciences Inc. Germantown, MD). RNA was quantified using Epoch Microplate Spectrophotometer (BioTek, Winooski, VT, USA).

\section{Reverse Transcription}

RNA from experiment 1 and 2 AMG tissue was reverse transcribed to complimentary DNA (cDNA) using 25ng of AMG RNA. RNA (25ng) from each sample was pooled to create a stock that was then serially diluted to create an 8-point standard curve to test the efficiency of the

primers used in this study. The SuperScript IV First-Strand Synthesis (Invitrogen, Waltham, MA, USA) was used to create cDNA. Samples were stored at $-20^{\circ} \mathrm{C}$ until use for qPCR testing within the manufacturers recommended storage time.

Another set of 25ng of AMG RNA was reverse transcribed using the Fluidigm Reverse Transcription Master Mix (Fluidigm, San Francisco, CA, USA) according to the manufacturer's 
instructions for all experiment 1 and 2 samples individually. RNA (25ng) from each sample in experiment 1 and 2 was pooled to create a stock that was then serially diluted to create an 8-point standard curve. The cDNA was then pre-amplified with 10 cycles using Fluidigm Pre-amp Master Mix and excess primers were removed via Exonuclease I digestion, per manufacturer's instructions. Pre-amplified samples were then diluted by $5 \mathrm{x}$ with $18 \mu \mathrm{L}$ TEKnova DNA Suspension Buffer (TEKnova, Hollister, CA, USA) and stored at $-20^{\circ} \mathrm{C}$ until use for qPCR testing within the manufacturers recommended storage time.

\section{Primer Design}

Primers for Quantitative real-time PCR analysis (RT-qPCR) were designed via the NCBI Primer-BLAST tool [44]. Using NCBI Gene tool [45], the mRNA sequences of a subset of DEGs from the RNAseq analysis [34] that were selected for validation (table 1) were searched using the most up to date version of the Macaca mulatta genome (Taxid ID: 9544, Mmul10). NCBI reference sequences for mRNA transcripts were selected from each gene and used as the PCR template in Primer-BLAST. Parameter search values that differed from the default were as follows: "PCR product size" was Min=90 and Max=200, "Organism" was Macaca mulatta (Taxid ID:9544), and “Allow splice variants" was selected. Genes which had no primer pair results in the last $25 \%$ of the coding region for the selected mRNA transcript were re-searched in Primer-BLAST. The new search parameters also included range "Forward primer: From" and "Reverse primer: To" values which spanned the last $25 \%$ of the coding region for said gene transcript.

In order to identify the most stable housekeeping genes for the amygdala, we selected and tested the stability of several commonly used housekeeping genes [46-50]: ACTG1, EIF4A2, $P G K 1, T O P 1, C Y C 1, G A P D H, R P L 13$, and UBE2D2. These genes were run in a qPCR to check 
for primer efficiencies and stability in the amygdala tissue. These primers sequences are listed in table 1 .

The DEGs selected for validation using microfluidic RT-qPCR (see below) were from the Cervera-Juanes et al. paper amyloid processing pathway (APP, PRKAR2A, CAPN1, PRKAR2B, CSNK2A1, CAPNS1, CSNK2A2, MAPK14, PRKCE, MAPT, MARK1, AKT3, PRKAR1A, MAPK, NCSTN). These gene primers are listed in table 2.

Quantitative real-time PCR analysis - QuantStudio

qPCR to test housekeeping genes stability and efficiency was performed using QuantStudio 12K Flex system (Applied Biosystems, Waltham, MA, USA). Samples and assays were prepared according to manufacturer's directions using PowerUp SYBR Green Master Mix (Applied Biosystems). Sample/Assay mixes were prepared with $6 \mu 1 \mathrm{SYBR}, 3 \mu 1$ water, $1 \mu 1$ forward and reverse primer at $10 \mu \mathrm{M}$, and $1 \mu \mathrm{l} \mathrm{cDNA}$ (concentration dependent on serial dilution sample used) per well in a 384-well plate. Sample/Assay mixes were then pipetted into a standard 384-well plate for thermocycling. Thermocycling qPCR conditions were as follows: $95^{\circ} \mathrm{C}$ for $60 \mathrm{~s}, 35$ cycles of $95^{\circ} \mathrm{C}$ for $5 \mathrm{~s}$ and $58^{\circ} \mathrm{C}$ for 20 s. Data was processed through automatic threshold for each assay, with derivative baseline correction using QuantStudio 12K Flex system (Applied Biosystems). The primer sequences are listed in Table 2.

\section{Microfluidic quantitative real-time PCR analysis - Fluidigm}

Following manufacturer's instructions, the sample and assay pre-mixes were prepared as previously described, then loaded into a 48.48 GE Dynamic Array IFC (Fluidigm) in duplicate assays using the Nanoflex IFC controller (Fluidigm). Thermocycling qPCR conditions were $95^{\circ} \mathrm{C}$ for $60 \mathrm{~s}, 35$ cycles of $95^{\circ} \mathrm{C}$ for $5 \mathrm{~s}$ and $58^{\circ} \mathrm{C}$ for $20 \mathrm{~s}$. Data was processed through automatic threshold for each assay. Then, derivative baseline correction was made using BioMark 
RealTime PCR Analysis Software 3.1.2 (Fluidigm) with quality threshold set at 0.65 . The primer sequences are listed in table 3.

Table 1 Genes selected for validation using RT-qPCR. Data taken from Cervera-Juanes et al. paper RNAseq analysis of the AMG amyloid processing pathway DEGs [34].

\begin{tabular}{|l|r|r|r|r|}
\hline Gene name & \multicolumn{1}{|c|}{ log FC } & \multicolumn{1}{c|}{ FC } & p-value & \multicolumn{1}{c|}{ fdr } \\
\hline AKT3 & -0.60 & 1.51 & $1.80 \mathrm{E}-02$ & $9.70 \mathrm{E}-02$ \\
\hline APP & -0.47 & 1.39 & $1.60 \mathrm{E}-02$ & $9.00 \mathrm{E}-02$ \\
\hline CAPN1 & -0.72 & 1.65 & $<0.0001$ & $2.00 \mathrm{E}-03$ \\
\hline CAPNS1 & -0.55 & 1.46 & $1.00 \mathrm{E}-03$ & $1.40 \mathrm{E}-02$ \\
\hline CSNK2A1 & -0.52 & 1.43 & $2.00 \mathrm{E}-03$ & $2.70 \mathrm{E}-02$ \\
\hline CSNK2A2 & -0.47 & 1.38 & $1.00 \mathrm{E}-02$ & $6.90 \mathrm{E}-02$ \\
\hline MAPK1 & -0.84 & 1.79 & $<0.0001$ & $2.00 \mathrm{E}-03$ \\
\hline MAPK14 & -0.83 & 1.78 & $4.00 \mathrm{E}-03$ & $4.20 \mathrm{E}-02$ \\
\hline MAPT & -0.56 & 1.48 & $1.70 \mathrm{E}-02$ & $9.50 \mathrm{E}-02$ \\
\hline MARK1 & -0.37 & 1.29 & $4.30 \mathrm{E}-02$ & $1.61 \mathrm{E}-01$ \\
\hline NCSTN & -0.59 & 1.50 & $<0.0001$ & $1.00 \mathrm{E}-02$ \\
\hline PRKAR1A & -0.52 & 1.44 & $3.00 \mathrm{E}-03$ & $3.60 \mathrm{E}-02$ \\
\hline PRKAR2A & -0.89 & 1.86 & $<0.0001$ & $1.00 \mathrm{E}-03$ \\
\hline PRKAR2B & -0.68 & 1.60 & $5.00 \mathrm{E}-03$ & $4.50 \mathrm{E}-02$ \\
\hline PRKCE & -0.49 & 1.40 & $4.00 \mathrm{E}-03$ & $3.0 \mathrm{E}-02$ \\
\hline
\end{tabular}

Table 2 Forward and reverse primers for the QuantStudio RT-qPCR housekeeping genes test for efficiency and reliability.

\begin{tabular}{|l|l|l|r|r|}
\hline Gene name & Forward primer (5' to $\left.\mathbf{3}^{\prime}\right)$ & Reverse primer (5' to $\left.\mathbf{3}^{\prime}\right)$ & $\begin{array}{l}\text { Amplicon } \\
\text { Size }\end{array}$ & $\begin{array}{l}\text { Annealing } \\
\left.\text { Temp (' }{ }^{\circ} \mathbf{C}\right)\end{array}$ \\
\hline CYC1 & GGCATGGTGGTGTAGGACTAC & TAGAGACCTTCCCGCAGTGA & 86 & 58 \\
\hline UBE2D2 & TGCCTGAGATTGCTCGGATCT & TCGCATACTTCTGAGTCCATTCC & 81 & 58 \\
\hline EIF4A2 & AATTCCGGTCAGGGTCAAGTC & GCCACACCTTTCCTCCCAAA & 166 & 58 \\
\hline TOP1 & GGCGAGTGAATCTAAGGATAATGAA & TGGATATCTTAAAGGGTACAGTGAA & 97 & 58 \\
\hline PGK1 & GCTGGACAAGCTGGATGTTA & GGCTCCATTGTCCAAGCAGA & 142 & 58 \\
\hline GAPDH & GGAAGCCCCATCACCATCTT & CGCCCCACTTGATTTTGG & 54 & 58 \\
\hline ACTG1 & AGCGCAAGTACTCAGTGTGG & CTACGCATCTGCTCAGTCCA & 149 & 58 \\
\hline RPL13 & AGCCTACGAGAAAGTTTGCCTAT & TCTTCTTCCTGTAGTGGATCTTGGC & 126 & 58 \\
\hline
\end{tabular}


Table 3 Forward and reverse primers used in the microfluidic RT- qPCR at $58^{\circ} \mathrm{C}$ annealing temperature. The sequences for GAPDH, ACTG1, and RPL13 are the same as those for table 1.

\begin{tabular}{|c|c|c|c|c|}
\hline Gene name & Forward primer (5' to $3^{\prime}$ ) & Reverse primer (5' to $3^{\prime}$ ) & $\begin{array}{l}\text { Amplicon } \\
\text { Size }\end{array}$ & $\begin{array}{l}\text { Annealing } \\
\text { Temp }\left({ }^{\circ} \mathrm{C}\right) \\
\end{array}$ \\
\hline$A P P$ & GACCACTCGACCAGGTTCTG & GCCCACCATGAGACCAATGA & 175 & 58 \\
\hline CAPN1 & TTTGACCTGGACAAGTCGGG & AACATGGTCTCTAGCCGCAC & 179 & 58 \\
\hline CAPNS1 & GGGTTCCACCTGAATGAGCA & CCTGGATTTGTCCAGTGCCA & 163 & 58 \\
\hline MAPK14 & TTGTTTCCTGGCACAGACCAT & TTCGGCATCTGCGTCAAAGA & 137 & 58 \\
\hline MAPT & CGTCAAGTCCAAGATCGGCT & CTCCCGGGACGTGTTTGATA & 143 & 58 \\
\hline MARK1 & CTGGCAGTACAACCCAGAGAG & CCCGTTTCATGGGATGGTGA & 187 & 58 \\
\hline PRKAR1A & GTGCTACAACGTCGGTCAGA & CTTAACGCACTTCAAGGGGC & 144 & 58 \\
\hline PRKAR2A & TGTGCCCCTCCTTAAATCGC & CTGGTTCCCACCATCCTTGT & 196 & 58 \\
\hline PRKAR2B & TCATTGCTCAGGGAGATTCGG & CTCCAAAGTACTGTCCCCGC & 147 & 58 \\
\hline CSNK2A1 & CGTCAGCAGCGCCAATATGA & TTGGCAGCAGCAATCACTGG & 96 & 58 \\
\hline CSNK2A2 & CAGCCCTGAGGCCCTAGAT & AAAGCACAGCATTGTCTGCAC & 146 & 58 \\
\hline PRKCE & TCAAACCGCGCATTAAAACCA & ATTGCTTCGTCCACAAGGGT & 59 & 58 \\
\hline AKT3 & GAGGGGAGTCATCATGAGCG & TGAGCCATCTGTCTTCAAAAGGA & 115 & 58 \\
\hline MAPK1 & CGTGACCTCAAGCCTTCCAA & CCCTGTACCAACGTGTAGCC & 139 & 58 \\
\hline NCSTN & СTTGTCTCCTGCCTTCGAACT & GGATGCCGAAGCCCACTATC & 149 & 58 \\
\hline GAPDH & GGAAGCCCCATCACCATCTT & CGCCCCACTTGATTTTGG & 54 & 58 \\
\hline ACTG1 & AGCGCAAGTACTCAGTGTGG & CTACGCATCTGCTCAGTCCA & 149 & 58 \\
\hline$R P L 13$ & AGCCTACGAGAAAGTTTGCCTAT & TCTTCTTCCTGTAGTGGATCTTGGC & 126 & 58 \\
\hline
\end{tabular}

\section{Results}

QuantStudio qPCR to select Housekeeping Gene(s) for the amygdala

From the QuantStudio results for each housekeeping gene, the average Ct values were graphed against the log of the RNA concentration of the standard curve to calculate the slope (a measure of the amplification efficiency). See figure 2 for the results of these graphs. The slopes for these genes: ACTG1, EIF4A2, PGK1, TOP1, CYC1, GAPDH, RPL13, UBE2D2 were -3.31, $3.34,-3.97,-3.44,-3.32,-3.21,-3.45,-3.48$, respectively. Thus, all of the primer pairs tested showed good efficiency. 
Standard deviation was also calculated for all $\mathrm{Ct}$ averages and ranged between $0.01-0.5$ per $\mathrm{Ct}$ average. The genes closest to a slope of -3.2 were automatically selected as housekeeping genes to use in further RT-qPCR tests (GAPDH and $A C T G 1)$. The third gene primer selected to use in further RT-qPCR tests was the gene with the closest slope to -3.2 and smallest amount of standard deviations within each point of its 8-point standard curve (RPL13). The three housekeeping genes identified (GAPDH, ACTG1 and RPL13) were then used as references genes in the microfluidic qPCR to validate the DEGs using samples from experiment 1 and experiment 2.

ACTG1

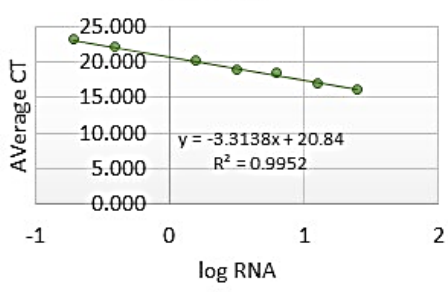

CYC1

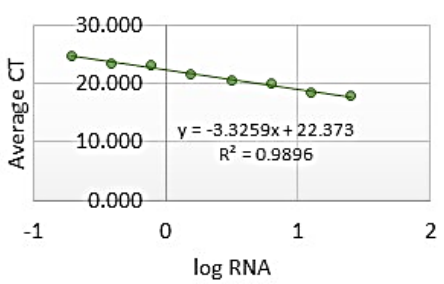

EIF4A2

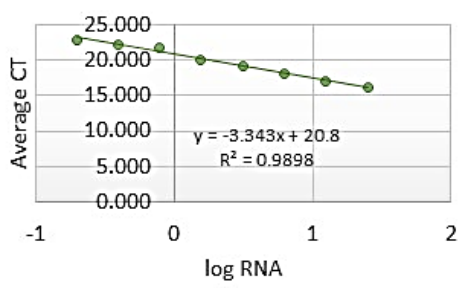

TOP1

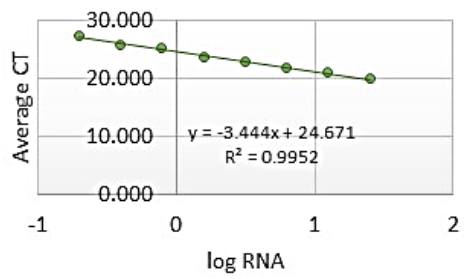

RPL13

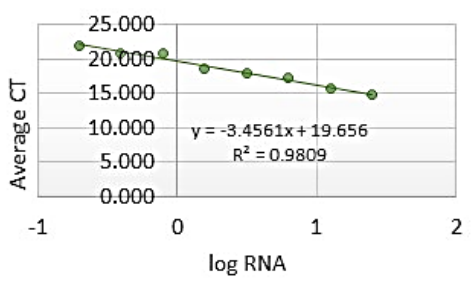

UBE2D2

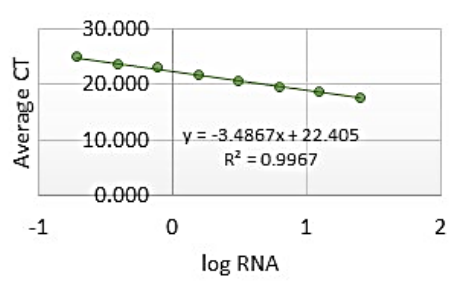

GAPDH

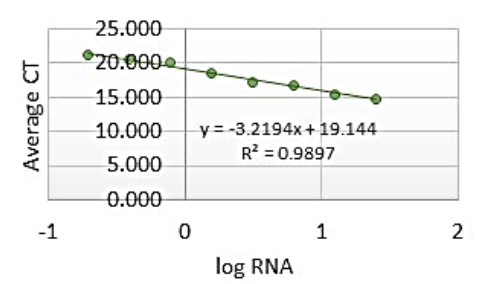

PGK1

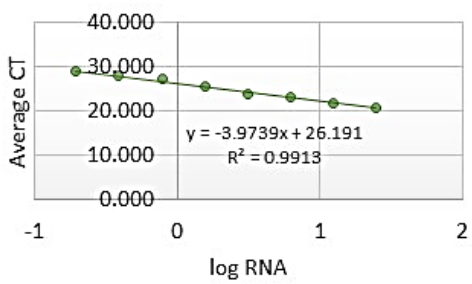

Figure 2 Efficiency graphs for the housekeeping genes tested using QuantStudio RT-qPCR with line of best fit equations and $\mathrm{R}^{2}$ values.

\section{Experiment 1 and 2 Data Processing}

We compared the expression levels between groups of the different housekeeping genes and selected the one(s) that showed no differences between treatment. For instance, RPL13 was used after it was confirmed the different experimental treatments did not alter its expression in the AMG between groups (independent t-test, $\mathrm{p}=0.79$ for experiment 1 and $\mathrm{p}=0.60$ for 
experiment 2). ACTG1 and GAPDH were found to be differentially expressed ( $\mathrm{p}=8.65 \mathrm{E}-03$ and $\mathrm{p}=1.59 \mathrm{E}-03$, respectively). Using independent $\mathrm{t}$-tests, differences in the mean expression between experimental groups respective to each experiment ( 1 or 2$)$ were analyzed (IBM SPSS Statistics; Armonk, NY, USA). Levene's test for homogeneity of variances was used before using the independent $t$-test. When the sample variance was heterogeneous $(\mathrm{p}>0.05)$, the Welch-Satterthwaite method was used to interpret the results of the SPSS t-test output. Experiment 1 Amyloid Processing Pathway

During the analysis of the subjects from experiment 1 , one subject was found to be an extreme outlier for all genes, determined by SPSS software. SPSS considers an extreme outlier if the data falls outside of the ranges: 3 rd quartile $+3 *$ interquartile range and 1 st quartile $3 *$ interquartile range. Based on this, the outlier was excluded from t-tests for all genes tested. Another sample was found to be an outlier for CSNK2Al and PRKAR2B, determined in SPSS by falling outside the ranges: 3 rd quartile $+1.5 *$ interquartile range and 1 st quartile -

$1.5 *$ interquartile range. The same sample was found to be an extreme outlier for $A P P$ and $M A P T$ (parameters identified previously). Because this sample was not consistent as an extreme outlier across all genes, it was left in for the t-test analysis.

From the previously identified genes in the amyloid processing pathway for the AMG, 15 genes were found to be differentially expressed $(\mathrm{p}<0.05)$ (figure 3a) using RT-qPCR analysis between the $\mathrm{OvH}$ and $\mathrm{OvH}-\mathrm{HT}$ females (samples from experiment 1). This was consistent with the data from the RNAseq findings (figure 3b). All of DEGs selected to test were significantly downregulated in the HT using RT-qPCR (table 4a), which was in agreement with the RNAseq findings (table $4 b)$. 


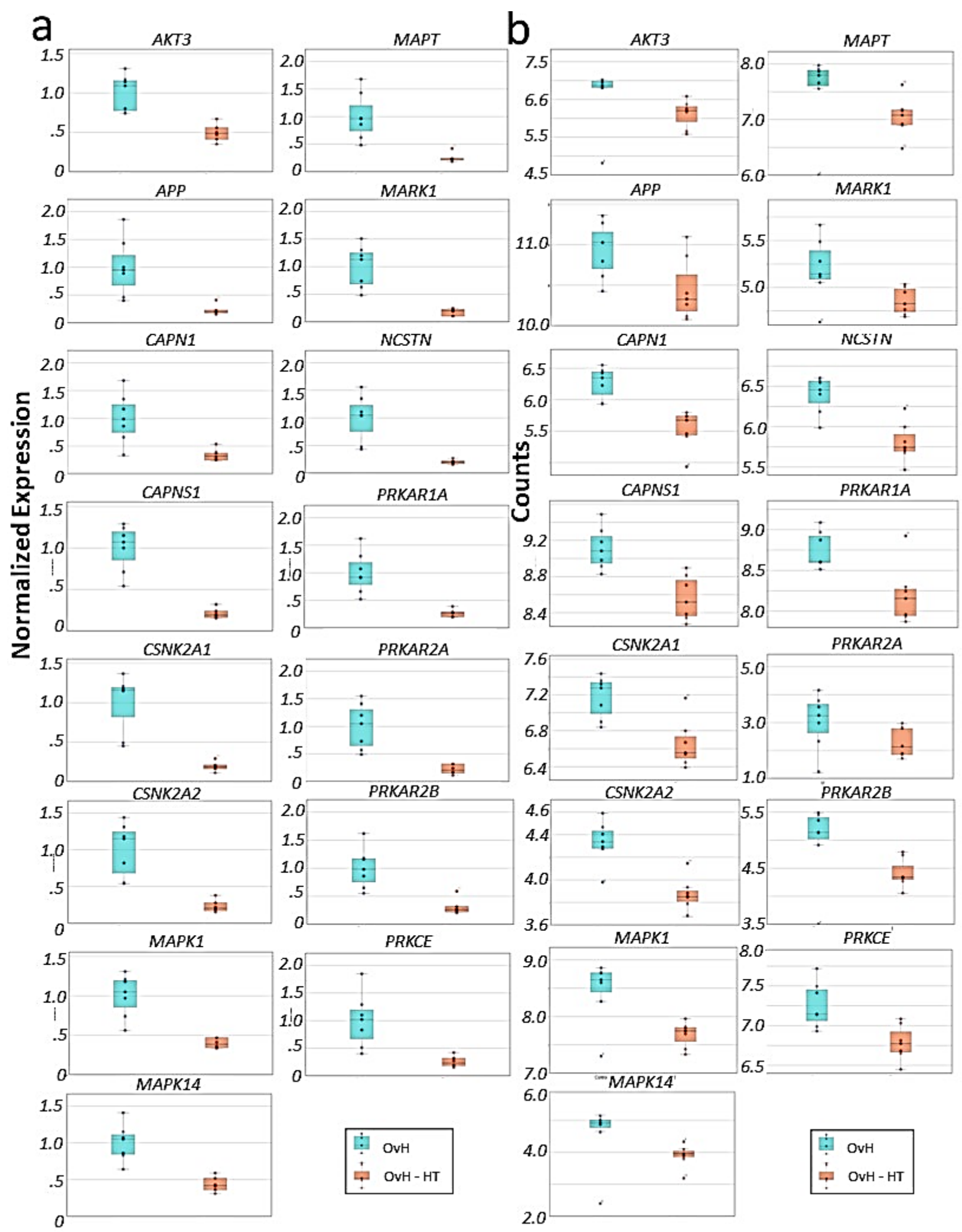

Figure 3 (a) Normalized expression boxplots from RT-qPCR data for experiment 1 with OvH vs OvH-HT rhesus macaque females. (b) Normalized counts boxplots from RNAseq data for experiment 1 with OvH vs OvH-HT rhesus macaque females, taken from Cervera-Juanes et al. paper [34]. 
Table 4 (a) Levene's Test for Homogeneity of Variances p-values and associated equal variance t-test or WelchSatterthwaite t-test p-values from experiment 1 RT-qPCR results from tested amyloid processing pathway genes. (b) Amyloid processing pathway genes selected for validation testing from Cervera-Juanes et al. paper [34] RNAseq data and their respective p-values.

\begin{tabular}{|c|c|c|}
\hline Gene name & $\begin{array}{l}\text { Levene's Test } \\
\text { p-value }\end{array}$ & p-value \\
\hline AKT3 & 0.012 & 6.42E-04 \\
\hline$A P P$ & 0.045 & 4.12E-03 \\
\hline CAPN1 & 0.037 & 7.41E-03 \\
\hline CAPNS1 & 0.026 & 1.97E-04 \\
\hline CSNK2A1 & 0.004 & 1.02E-03 \\
\hline CSNK2A2 & 0.002 & 1.11E-03 \\
\hline MAPK1 & 0.024 & 7.84E-04 \\
\hline МАРК 14 & 0.089 & 3.24E-04 \\
\hline MAPT & 0.031 & 3.14E-03 \\
\hline MARK1 & 0.001 & 1.16E-03 \\
\hline NCSTN & 0.018 & 2.27E-03 \\
\hline PRKAR1A & 0.025 & 1.80E-03 \\
\hline PRKAR2A & 0.004 & $2.13 \mathrm{E}-03$ \\
\hline PRKAR2B & 0.084 & 1.12E-03 \\
\hline PRKCE & 0.047 & 3.97E-03 \\
\hline
\end{tabular}

b
\begin{tabular}{|l|r|}
\hline Gene name & p-value \\
\hline AKT3 & $1.80 \mathrm{E}-02$ \\
\hline APP & $1.60 \mathrm{E}-02$ \\
\hline CAPN1 & $<0.0001$ \\
\hline CAPNS1 & $1.00 \mathrm{E}-03$ \\
\hline CSNK2A1 & $2.00 \mathrm{E}-03$ \\
\hline CSNK2A2 & $1.00 \mathrm{E}-02$ \\
\hline MAPK1 & $<0.0001$ \\
\hline MAPK14 & $4.00 \mathrm{E}-03$ \\
\hline MAPT & $1.70 \mathrm{E}-02$ \\
\hline MARK1 & $4.30 \mathrm{E}-02$ \\
\hline NCSTN & $<0.0001$ \\
\hline PRKAR1A & $3.00 \mathrm{E}-03$ \\
\hline PRKAR2A & $<0.0001$ \\
\hline PRKAR2B & $5.00 \mathrm{E}-03$ \\
\hline PRKCE & $4.00 \mathrm{E}-03$ \\
\hline
\end{tabular}

Experiment 2 Amyloid Processing Pathway

Using the genes identified as DEGs for experiment 1, the same primers were tested on the samples from experiment 2 between old and young females to determine if any of these effects could be seen with age under a regular diet. SPSS identified one outlier for APP outside the ranges of: 3 rd quartile $+1.5 *$ interquartile range and 1 st quartile $-1.5 *$ interquartile range and two significant outliers for MAPK14. These outliers were not excluded because they were not consistent in across other genes, suggesting they are accurate values for experiment 2 . Of the 
genes involved in the amyloid processing pathway that were selected for testing with RT-qPCR, none of them were found to be significant $(\mathrm{p}<0.05)$ for experiment 2 (figure $4 a \& b$ ). 


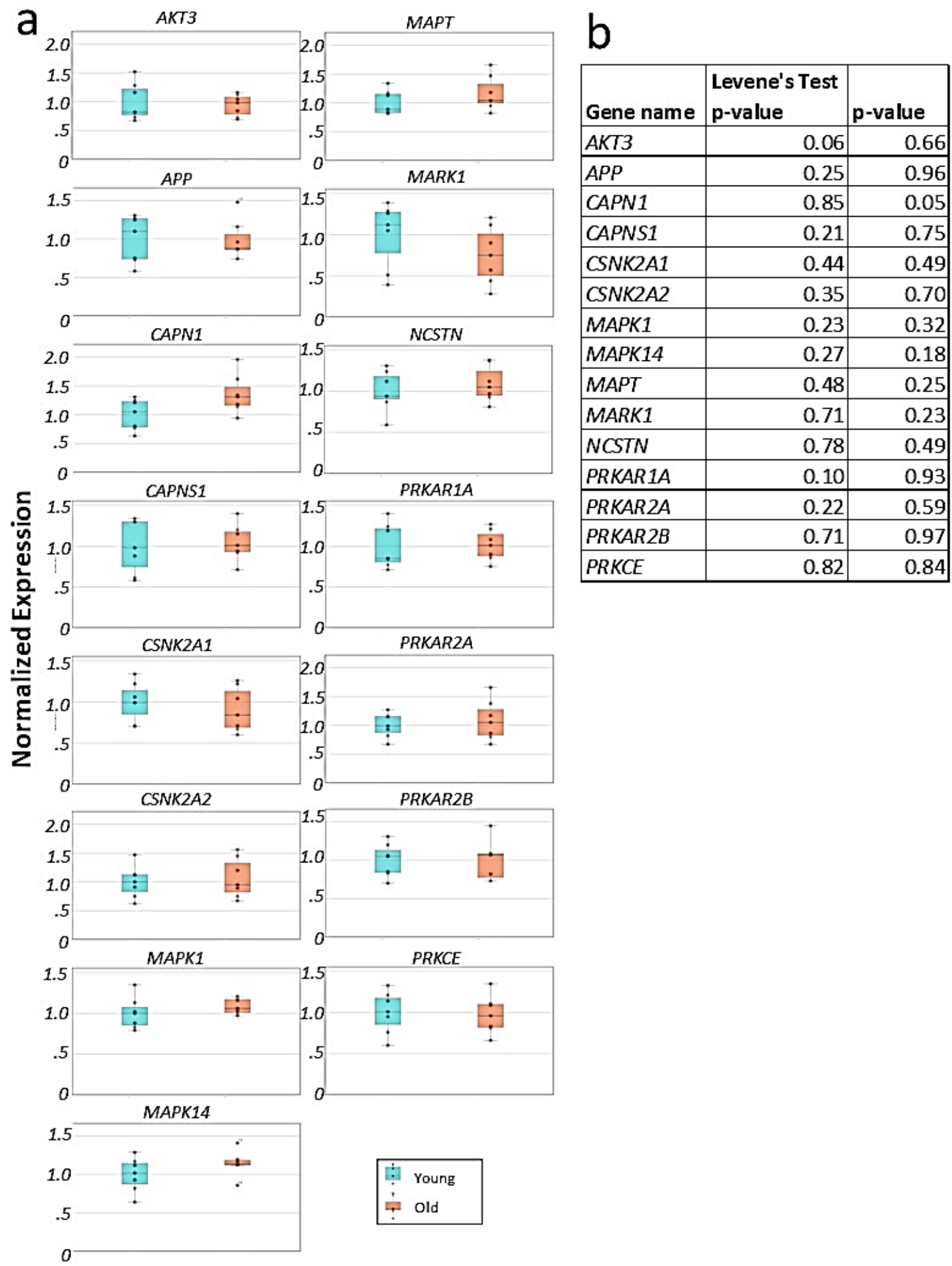

Figure 4 (a) Normalized expression boxplots from RT-qPCR data for experiment 2 with young vs old rhesus macaque females. (b) Table with Levene's Test for Homogeneity of Variances p-values and associated equal variance $\mathrm{t}$-test or Welch-Satterthwaite t-test $\mathrm{p}$-values from experiment $2 \mathrm{RT}$-qPCR results from tested amyloid processing pathway genes. 


\section{Discussion}

The purpose of this study was to confirm the results from the Cevera-Juanes et al. paper and validate the RNAseq findings from that study using the same AMG RNA samples. We were able to validate the RNAseq data because of the 15 genes selected for validation using RT-qPCR, all of them were verified to be significantly different. More specifically, the selected genes were also downregulated in the HT group, same as the RNAseq data [34].

Of the genes selected for validation testing, the RNAseq data had counts per million as low as 2.3 mean logCPM per experimental group for experiment 1 [34]. However, using this low cutoff value does not guarantee that all genes at that level will be differentially expressed. With the qPCR findings confirming the genes at $2.3 \log C P M$ were differentially expressed, it allows us to say with confidence that using a cutoff of $2.3 \log C P M$ in the future is valid for this type of dataset, and the genes at this level will be differentially expressed.

The amyloid processing pathway is responsible for the proteolytic cleavage of the amyloid precursor protein (APP) to A $\beta$ peptide, which has been associated with AD [51-52]. The genes APP (amyloid precursor protein), NCSTN (nicastrin), and MAPT (microtubule associated protein tau) are all involved in the generation of A $\beta$. During amyloid processing, APP is cleaved by the $\gamma$-secretase complex, which contains the protein encoded by NCSTN [53]. Coincidentally, the findings of this study showed that these genes, along with the rest of selected genes for validation are downregulated with HT in females under a chronic WSD.

Additional genes that have been implicated in the AD pathology, MARK, AKT, MARK, and MAPT [34,54] through their contributions to developing neuritic plaques and neurofibrillary tangles were also investigated in this study. Many neurodegenerative diseases are characterized by the abnormal clustering of tau in neurons or glia, including AD, and MAPT is a principal 
component of these tau tangles [54]. With A $\beta$ plaques and neurofibrillary tangles being indicators of AD development $[42,54]$, our results could indicate that chronic WSD may increase risk for $\mathrm{AD}$, and that $\mathrm{HT}$ in these individuals could have a down-regulative effect on the pathway that synthesizes $A \beta$ and tau, indicating positive cognitive benefits of HT use on menopausal women on WSD. This correlates with other studies which have found neuroprotective effects of estrogen HTs on women in early menopause such as protecting neurons against beta-amyloid toxicity and calcium toxicity [55-56]. Our results in rhesus macaques contribute to this evidence and the use of HT at menopause onset, while exercising caution that long-term WSD could be causing increased risk of AD and decreasing the effectiveness of HT treatments.

Although there are important differences in experimental design between the subjects included in experiment 1 and experiment 2, we included experiment 2 in this validation study to determine if alterations in the amyloid pathway naturally occur with age, independently of diet and estrogen levels. With no significant differences in gene expression observed in genes within the amyloid processing pathway for experiment 2 subjects, we can conclude there are no significant expression changes in the amygdala between young rhesus macaque pre-menopausal females and post-menopausal females on a SD. But, as previously established, WSD is a common factor contributing to obesity worldwide [28], so the genetic link between increased neurodegeneration and menopause suggested could have more to do with WSD and obesity than age $[5,9]$. This is supported by other studies which indicate a strong connection between obesity and impaired cognitive function, including increased risk of neurodegenerative diseases like AD [57-59]. 
We can infer from these two experiments that the WSD during lack of estrogens is a strong risk factor for neurodegenerative risk in females. The downregulation of the amyloid processing genes indicates that there may be an insulating or neuroprotective effect from HT treatment, though without further research, it is unclear if WSD is exacerbating the condition in the absence of HT. We are currently conducting a similar study, analyzing the effects of HT in OvH females under a regular diet. This study will further clarify the risk of WSD for AD.

Future directions for this research would be to continue research on HT and WSD correlations for the amyloid processing pathway genes. We are planning to use immunohistochemistry (IHC) and Western blots to further visualize the changes in the amyloid processing pathway of the AMG. Through IHC and Western blots of this tissue, we will be able to see if the changes in gene expression correlate to proteomic changes. These findings along with future studies which also account for diet type and $\mathrm{OvH}$ vs non-OvH, would then allow relative expression values to be compared across experiments and result in more definitive findings about what is causing downregulation of the amyloid processing pathway.

\section{Funding}

This work was supported by National Institutes of Health grants AG-029612, AG-062220, OD011092, and OD-011895. 


\section{References}

[1] R. D. Brinton, J. Yao, F. Yin, W. J. Mack, and E. Cadenas, "Perimenopause as a neurological transition state," Nat. Rev. Endocrinol., vol. 11, no. 7, pp. 393-405, 2015.

[2] J. L. Downs and P. M. Wise, "The role of the brain in female reproductive aging," Mol. Cell. Endocrinol., vol. 299, no. 1, pp. 32-38, 2009.

[3] C. E. Finch, "The menopause and aging, a comparative perspective," J. Steroid Biochem. Mol. Biol., vol. 142, pp. 132-141, 2014.

[4] L. S. Cohen, C. N. Soares, and H. Joffe, "Diagnosis and management of mood disorders during the menopausal transition," Am. J. Med., vol. 118 Suppl 12B, no. 12, pp. 93-97, 2005.

[5] O. Scheyer et al., "Female sex and Alzheimer's risk: The menopause connection," J. Prev. Alzheimers Dis., pp. 1-6, 2018.

[6] J. E. Manson et al., "Menopausal hormone therapy and long-term all-cause and causespecific mortality: The Women's Health Initiative randomized trials," JAMA, vol. 318, no. 10, pp. 927-938, 2017.

[7] "Considering sex and gender in Alzheimer disease and other dementias," Dialogues Clin Neurosci, vol. 18, no. 4, pp. 437-446, 2016.

[8] E. R. Bacon, A. Mishra, Y. Wang, M. K. Desai, F. Yin, and R. D. Brinton, "Neuroendocrine aging precedes perimenopause and is regulated by DNA methylation," Neurobiol. Aging, vol. 74, pp. 213-224, 2019.

[9] D. A. Davey, "Alzheimer'S disease, dementia, mild cognitive impairment and the menopause: A 'window of opportunity'?," Womens. Health (Lond. Engl.), vol. 9, no. 3, pp. 279-290, 2013.

[10] Writing Group for the Women's Health Initiative Investigators, "Risks and benefits of estrogen plus progestin in healthy postmenopausal women: Principal results from the women's health initiative randomized controlled trial," JAMA, vol. 288, no. 3, pp. 321-333, 2002.

[11] F. Lizcano and G. Guzmán, "Estrogen Deficiency and the Origin of Obesity during Menopause," Biomed Res. Int., vol. 2014, p. 757461, 2014.

[12] A. Flöter, J. Nathorst-Böös, K. Carlström, and B. von Schoultz, "Addition of testosterone to estrogen replacement therapy in oophorectomized women: effects on sexuality and wellbeing," Climacteric, vol. 5, no. 4, pp. 357-365, 2002.

[13] J. Munoz, A. Derstine, and B. A. Gower, "Fat distribution and insulin sensitivity in postmenopausal women: Influence of hormone replacement," Obes. Res., vol. 10, no. 6, pp. 424-431, 2002.

[14] J. Marjoribanks, C. Farquhar, H. Roberts, A. Lethaby, and J. Lee, "Long-term hormone therapy for perimenopausal and postmenopausal women," Cochrane Database Syst. Rev., vol. 1, no. 1, p. CD004143, 2017.

[15] M. L. Walker and J. G. Herndon, "Menopause in nonhuman primates?," Biol. Reprod., vol. 79, no. 3, pp. 398-406, 2008.

[16] O. Scheyer et al., "Female sex and Alzheimer's risk: The menopause connection," J. Prev. Alzheimers Dis., vol. 5, no. 4, pp. 225-230, 2018. 
[17] S. G. Kohama et al., "Effect of ovarian hormone therapy on cognition in the aged female rhesus macaque," J. Neurosci., vol. 36, no. 40, pp. 10416-10424, 2016.

[18] A. Paganini-Hill and V. W. Henderson, "Estrogen deficiency and risk of Alzheimer's disease in women," Am. J. Epidemiol., vol. 140, no. 3, pp. 256-261, 1994.

[19] S. R. Rapp et al., "Effect of estrogen plus progestin on global cognitive function in postmenopausal women: the Women's Health Initiative Memory Study: a randomized controlled trial: The women's health initiative memory study: A randomized controlled trial," JAMA, vol. 289, no. 20, pp. 2663-2672, 2003.

[20] W. A. Rocca, B. R. Grossardt, and L. T. Shuster, "Oophorectomy, estrogen, and dementia: a 2014 update," Mol. Cell. Endocrinol., vol. 389, no. 1-2, pp. 7-12, 2014.

[21] H. Shao et al., "Hormone therapy and Alzheimer disease dementia: new findings from the Cache County Study," Neurology, vol. 79, no. 18, pp. 1846-1852, 2012.

[22] J. Kozakowski, M. Gietka-Czernel, D. Leszczynska, and A. Majos, "Obesity in menopause our negligence or an unfortunate inevitability? Prz Menopauzalny.” 2017.

[23] R. Pacifici, "Estrogen deficiency, T cells and bone loss," Cell. Immunol., vol. 252, no. 1-2, pp. 68-80, 2008.

[24] S. D. Sullivan, P. M. Sarrel, and L. M. Nelson, "Hormone replacement therapy in young women with primary ovarian insufficiency and early menopause," Fertil. Steril., vol. 106, no. 7, pp. 1588-1599, 2016.

[25] Z. Tauqeer, G. Gomez, and F. C. Stanford, "Obesity in women: Insights for the clinician," J. Womens. Health (Larchmt), vol. 27, no. 4, pp. 444-457, 2018.

[26] "Centers for Disease Control and Prevention. Overweight \& Obesity," Cdc.gov, Jun-2020. [Online]. Available: https://www.cdc.gov/obesity/data/adult.html. [Accessed: 15-Nov-2021].

[27] Craig M. Hales MDC, Cheryl D. Fryar, and Cynthia L. Ogden, "Prevalence of Obesity and Severe Obesity Among Adults: United States," 2017-2018. [Online]. Available: https://wwwcdcgov/nchs/products/databriefs/db360htm. [Accessed: 15-Nov-2021].

[28] A. Jayaraman and C. J. Pike, "Alzheimer's disease and type 2 diabetes: Multiple mechanisms contribute to interactions," Curr. Diab. Rep., vol. 14, no. 4, 2014.

[29] G. K. Gouras, D. Tampellini, R. H. Takahashi, and E. Capetillo-Zarate, "Intraneuronal betaamyloid accumulation and synapse pathology in Alzheimer's disease," Acta Neuropathol., vol. 119, no. 5, pp. 523-541, 2010.

[30] A. Christensen and C. J. Pike, "Age-dependent regulation of obesity and Alzheimer-related outcomes by hormone therapy in female 3xTg-AD mice," PLoS One, vol. 12, no. 6, p. e0178490, 2017.

[31] H. N. Polotsky and A. J. Polotsky, "Metabolic implications of menopause," Semin. Reprod. Med., vol. 28, no. 5, pp. 426-434, 2010.

[32] B. Zimmerman et al., "Longitudinal effects of immediate and delayed estradiol on cognitive performance in a spatial maze and hippocampal volume in menopausal macaques under an obesogenic diet," Front. Neurol., vol. 11, p. 539, 2020.

[33] A. Rehman, A. K. Y. Neuroanatomy, and O. Lobe, "StatPearls." .

[34] R. Cervera-Juanes, P. Darakjian, M. Ball, S. G. Kohama, and H. F. Urbanski, "Effects of estradiol supplementation on the brain transcriptome of old rhesus macaques maintained on an obesogenic diet," GeroScience, 2021. 
[35] S. M. Stufflebeam and B. R. Rosen, "Mapping cognitive function," Neuroimaging Clin. N. Am., vol. 17, no. 4, pp. 469-84, viii-ix, 2007.

[36] S. Funahashi and J. M. Andreau, "Prefrontal cortex and neural mechanisms of executive function," J. Physiol. Paris, vol. 107, no. 6, pp. 471-482, 2013.

[37] K. S. Anand and V. Dhikav, "Hippocampus in health and disease: An overview," Ann. Indian Acad. Neurol., vol. 15, no. 4, pp. 239-246, 2012.

[38] L. Bonnet, A. Comte, L. Tatu, J.-L. Millot, T. Moulin, and E. Medeiros de Bustos, "The role of the amygdala in the perception of positive emotions: an "intensity detector," Front. Behav. Neurosci., vol. 9, p. 178, 2015.

[39] K. J. Ressler, “Amygdala activity, fear, and anxiety: Modulation by stress," Biol. Psychiatry, vol. 67, no. 12, pp. 1117-1119, 2010.

[40] E. Yavas, S. Gonzalez, and M. S. Fanselow, "Interactions between the hippocampus, prefrontal cortex, and amygdala support complex learning and memory," F1000Res., vol. 8, p. $1292,2019$.

[41] M. P. Murphy and H. LeVine 3rd, "Alzheimer's disease and the amyloid-beta peptide," J. Alzheimers. Dis., vol. 19, no. 1, pp. 311-323, 2010.

[42] V. J. De-Paula, M. Radanovic, B. S. Diniz, and O. V. Forlenza, “Alzheimer's Disease,” in Protein Aggregation and Fibrillogenesis in Cerebral and Systemic Amyloid Disease, Dordrecht: Springer Netherlands, 2012, pp. 329-352.

[43] M. D. Robinson, D. J. McCarthy, and G. K. Smyth, "edgeR: a Bioconductor package for differential expression analysis of digital gene expression data," Bioinformatics, vol. 26, no. 1, pp. 139-140, 2010.

[44] J. Ye, G. Coulouris, I. Zaretskaya, I. Cutcutache, S. Rozen, and T. L. Madden, "PrimerBLAST: a tool to design target-specific primers for polymerase chain reaction," BMC Bioinformatics, vol. 13, no. 1, p. 134, 2012.

[45] "Home - Gene - NCBI," Nih.gov, National Library of Medicine (US), National Center for Biotechnology Information. [Online]. Available: https://www.ncbi.nlm.nih.gov/gene/. [Accessed: 10-Aug-2021].

[46] L.-N. Schuhmacher and E. S. J. Smith, "Expression of acid-sensing ion channels and selection of reference genes in mouse and naked mole rat," Mol. Brain, vol. 9, no. 1, 2016.

[47] V. L. Harbour, Y. Weigl, B. Robinson, and S. Amir, "Phase differences in expression of circadian clock genes in the central nucleus of the amygdala, dentate gyrus, and suprachiasmatic nucleus in the rat," PLoS One, vol. 9, no. 7, p. e103309, 2014.

[48] V. Leduc, V. Legault, D. Dea, and J. Poirier, "Normalization of gene expression using SYBR green qPCR: A case for paraoxonase 1 and 2 in Alzheimer's disease brains," $J$. Neurosci. Methods, vol. 200, no. 1, pp. 14-19, 2011.

[49] A. Kouli, M. Camacho, K. Allinson, and C. H. Williams-Gray, "Neuroinflammation and protein pathology in Parkinson's disease dementia," Acta Neuropathol. Commun., vol. 8, no. 1, p. 211, 2020.

[50] F. Mauvais-Jarvis, D. J. Clegg, and A. L. Hevener, "The role of estrogens in control of energy balance and glucose homeostasis," Endocr. Rev., vol. 34, no. 3, pp. 309-338, 2013.

[51] H. Xu, R. Wang, Y.-W. Zhang, and X. Zhang, "Estrogen, beta-amyloid metabolism/trafficking, and Alzheimer's disease," Ann. N. Y. Acad. Sci., vol. 1089, no. 1, pp. 324-342, 2006. 
[52] H. M. Wilkins and R. H. Swerdlow, "Amyloid precursor protein processing and bioenergetics," Brain Res. Bull., vol. 133, pp. 71-79, 2017.

[53] K. H. Strang, T. E. Golde, and B. I. Giasson, "MAPT mutations, tauopathy, and mechanisms of neurodegeneration," Lab. Invest., vol. 99, no. 7, pp. 912-928, 2019.

[54] S. Pugazhenthi, L. Qin, and P. H. Reddy, "Common neurodegenerative pathways in obesity, diabetes, and Alzheimer's disease," Biochim. Biophys. Acta Mol. Basis Dis., vol. 1863, no. 5, pp. 1037-1045, 2017.

[55] Y. Hara et al., "Estrogen alters the synaptic distribution of phospho-GluN2B in the dorsolateral prefrontal cortex while promoting working memory in aged rhesus monkeys," Neuroscience, vol. 394, pp. 303-315, 2018.

[56] J.-P. Brion, "Neurofibrillary tangles and Alzheimer's disease," Eur. Neurol., vol. 40, no. 3, pp. 130-140, 1998.

[57] L. Dye, N. B. Boyle, C. Champ, and C. Lawton, "The relationship between obesity and cognitive health and decline," Proc. Nutr. Soc., vol. 76, no. 4, pp. 443-454, 2017.

[58] P. S. Green, K. E. Gridley, and J. W. Simpkins, "Estradiol protects against beta-amyloid (25-35)-induced toxicity in SK-N-SH human neuroblastoma cells," Neurosci. Lett., vol. 218, no. 3, pp. 165-168, 1996.

[59] J. Nilsen, S. Chen, R. W. Irwin, S. Iwamoto, and R. D. Brinton, "Estrogen protects neuronal cells from amyloid betainduced apoptosis via regulation of mitochondrial proteins and function," BMC Neurosci, vol. 7, no. 74, 2006. 